\title{
Percutaneous transluminal angioplasty and stenting for innominate artery pre-occlusive stenosis with subclavian steal syndrome - case report and current challenges
}

\author{
Bogdan Casaru', Athena Ribigan', Bogdan Dorobat ${ }^{2}$, Florina Antochi ${ }^{1}$ \\ ${ }^{1}$ Neurology Department, University Emergency Hospital, Bucharest, Romania \\ ${ }^{2}$ Interventional Radiology Departement, University Emergency Hospital, Bucharest, Romania
}

\begin{abstract}
Introduction. Atherosclerotic occlusive disease of the innominate artery (IA) represents a rare entity, comprising less than $2 \%$ of all extracranial causes of altered cerebral blood flow. This type of lesion is associated with adaptative hemodynamic changes increasing the risk of developing both cerebral and upper extremity ischaemia. Subclavian steal syndrome is a serious complication that may occur with occlusive lesions of the innominate artery and may represent an indication for revascularization.

Case presentation. In the current paper we present the case of a patient who was admitted in our clinic for further investigations regarding episodes of dizziness and a difference of $30 \mathrm{mmHg}$ in the systolic blood pressure between his arms. On duplex ultrasonography scanning, an IA stenosis suspicion was raised, with associated retrograde flow through the right vertebral artery (VA) at light efforts of the right upper extremity. An angiographic evaluation confirmed the sub-occlusive lesion and reversed flow through the right VA. A decision was reached to undertake percutaneous transluminal angioplasty and stenting. There was no report of periprocedural complications, the intervention has been considered successful with no restenosis and no neurologic symptoms at 9 months follow-up.

Discussion. Until recently, surgical treatment was the only option in the management of atherosclerotic disease of the IA, a procedure with high complication, morbidity and mortality rate. Therefore, endovascular treatment gained more ground and is considered today as the first line of treatment for this type of lesion.

Conclusion. PTA and stenting of the IA still remains challenging mainly due to complex anatomy and the rarity of this pathology and we must keep in mind that the decision for the revascularization technique is personalized. This present case brings new evidence that percutaneous transluminal angioplasty is a safe and efficient method to manage an atherosclerotic lesion of the brachiocephalic artery.
\end{abstract}

Keywords: innominate artery stenosis, subclavian steal syndrome, percutaneous angioplasty and stenting

\section{INTRODUCTION}

Atherosclerotic occlusive disease of the innominate artery (IA) represents a rare entity, comprising less than $2 \%$ of all extracranial causes of altered cerebral blood flow (1). This type of lesion may result in a number of adaptative hemodynamic changes, increasing the risk of developing both cerebral and upper extremity ischaemia. Subclavian steal syndrome is a serious complication that may occur with occlusive lesions of the innominate artery and may represent an indication for revascular- ization. We hereby would like to present the case of a patient admitted in our clinic for this pathology and for whom the decision of percutaneous transluminal angioplasty and stenting has been made.

\section{CASE PRESENTATION}

A 62-year old male patient with a history of dyslipidemia, impaired glucose tolerance and 30 years of cigarette smoking, was referred to the Neurology Department of the Emergency University Hospital Bucharest for further investigations regarding a dif- 
ference of $30 \mathrm{mmHg}$ in the systolic blood pressure between arms. Clinical examination was normal, with the only complaints being frequent episodes of dizziness and bilateral tinnitus, symptoms which could not be associated with excessive use of the right arm where the blood pressure was lower.

The first diagnostic step was duplex ultrasonography (Fig. 1), which revealed post-stenotic velocities and waveforms at the level of the right common carotid artery (CCA) and subclavian artery (SA), an aspect suggestive of a lesion at the level of the IA. Furthermore, the flow through the right ver-
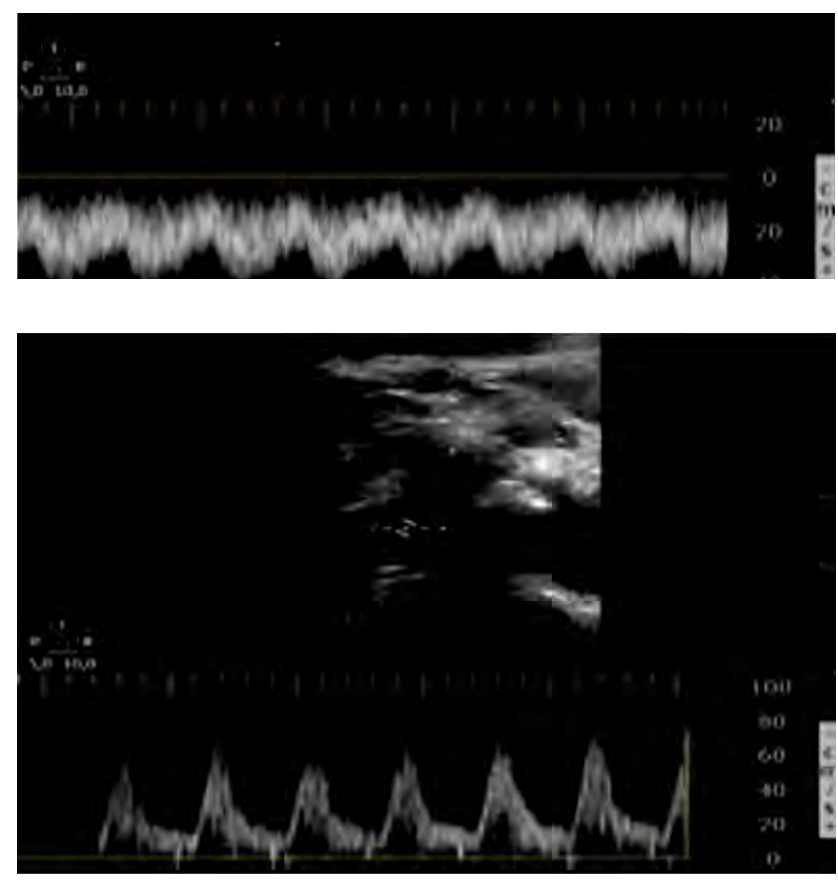

FIGURE 1. Top left: right CCA with decreased velocities, dampened waveform. Bottom left: right SA without triphasic waveform typical of high resistance extremity artery. Top right: right $V A$ with normal anterograde flow. Bottom right: right VA with retrograde flow after physical exercises with the right hand.
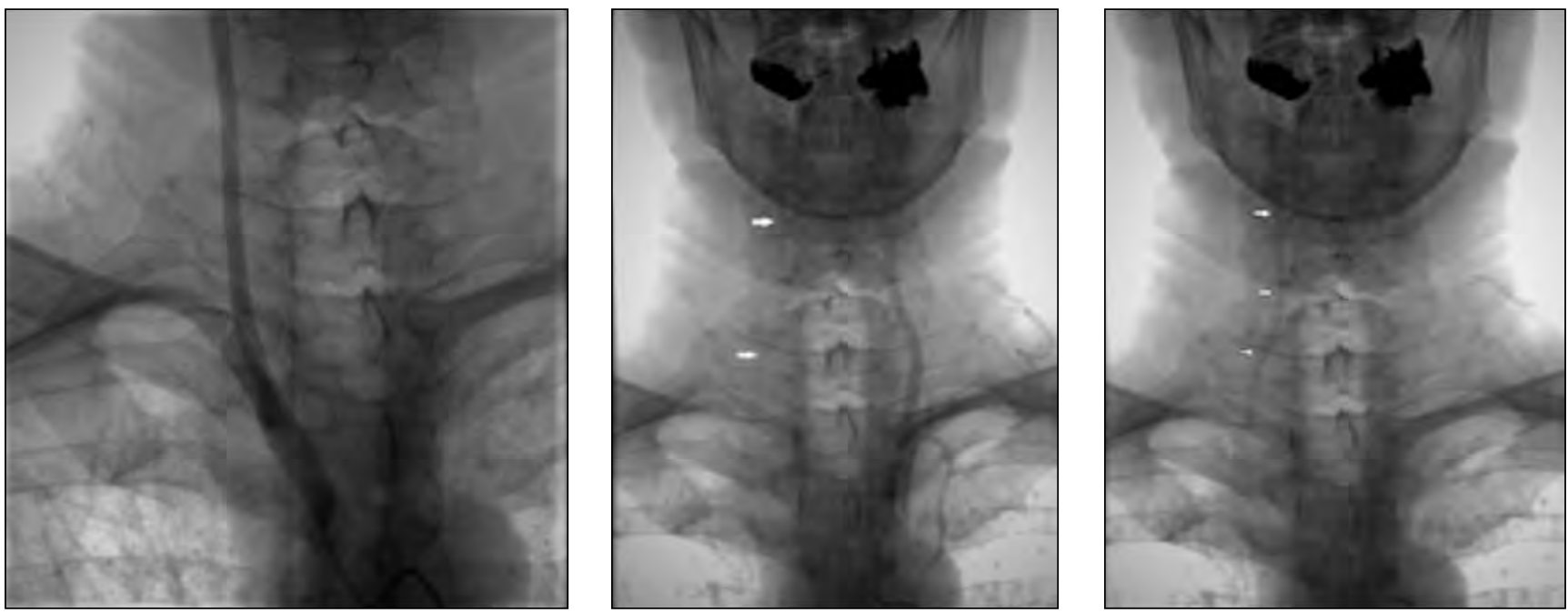

FIGURE 2. Left: sub-occlusive stenosis at the origin of the IA with no anterograde flow through the right VA. Middle and right: dye injection at the level of the left SA with tardive retrograde filling of the right VA, confirming the subclavian steal syndrome on the right side. 


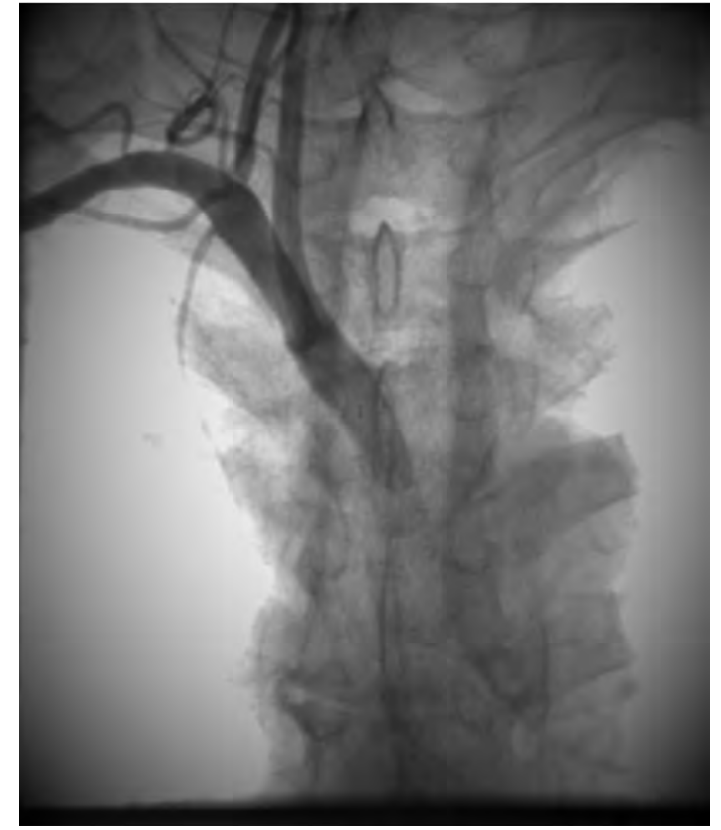

FIGURE 3. Stent deployment with no residual stenosis and anterograde flow in the right VA
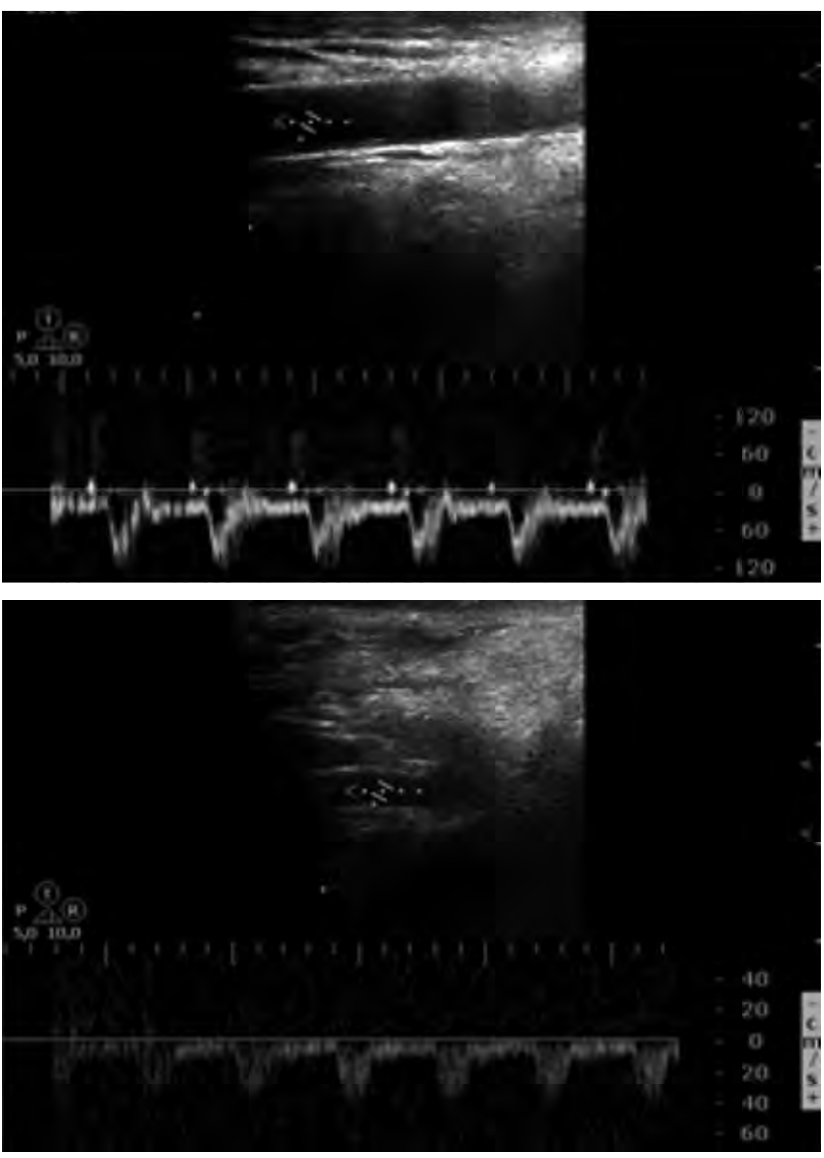

FIGURE 4. Ultrasonography at 9 months follow-up revealed normal velocities and waveforms at the level of the right CCA (Top) and anterograde flow through the right $V A$ even after hand exercises (Bottom)

After informing the patient about all the treatment options, a common decision was reached for percutaneous transluminal angioplasty and stent- ing. A retrograde brachial approach was chosen, using a 0.035 inch guidewire to cross the stenotic region and then a Visi-Pro 9x27 $\mathrm{mm}$ Bare Metal Stent (BMS) was deployed (Fig. 3). There were no images of residual stenosis described and the procedure underwent with no periprocedural incidents. Patient was discharged 5 days after the intervention, asymptomatic, with equal blood pressure between arms, anterograde flow through the right VA and normal velocities of the right CCA. He received double antiplatelet therapy and statin throughout his admission, and it was recommended to continue this treatment until the next evaluation. At 9-months follow-up the patient was free of symptoms, no difference in blood pressure between arms was noted and there were no indirect signs of intra-stent stenosis on duplex sonography (Fig. 4).

\section{DISCUSSION}

Treatment of atheromatous lesions of the IA has greatly evolved over the last 4 decades. What used to be achieved by open surgery either using a trans-thoracic or extra-thoracic route at a high cost in terms of mortality and morbidity $(2,3)$, benefits nowadays from an endovascular procedure or a hybrid approach. Standard open surgical procedures are now mostly reserved for extensive multivessel involvement or after failed endovascular procedures. Recently, they have been replaced by minimally invasive options with different antegrade and retrograde endovascular catheter-based approaches and combinations with surgical exposure of the CCA. The 2017 ESVS Guidelines on the Management of Atherosclerotic Carotid and Vertebral Artery Disease does not recommend revascularization in asymptomatic patients and most proximal CCA and IA stenoses should be considered for treatment via open retrograde angioplasty and stenting (IIa) (4).

Even though the natural course of the disease is still unknown, stenting is considered now the first line of treatment for symptomatic patients, including the ones with subclavian steal syndrome (5). Its efficacy has been validated in follow-up studies, which showed a low complication rate and a high patency rate at least on the short and mid-term $(6,7,8)$. Current studies are trying to identify safety, efficacy and outcome of the stent placing procedures, focusing on different technical approaches. 
One team from Bern (9) pointed out some aspects that might be helpful in making the decision on what type of intervention is best suited for every patient. From a total of 18 symptomatic patients with high-grade stenosis ( $>80 \%$ ) of the IA, 11 were treated by percutaneous stenting and for 7 patients the lesion was accessed retrogradely through a cervical cut-down with common carotid arteriotomy. They reported a technical success rate of $94.4 \%$, a procedure-related transient complication rate of $11.1 \%$ and excellent or good clinical outcome according to a mRS score of 0 or 1 , with restenosis of 2 lesions at a mean follow-up of 2.7 years. Their approach was different based on degree of stenosis, amount of calcified plaques, anatomy of the IA branching from the aortic arch and the presence of a high-grade tandem stenosis at the level of carotid bifurcation. They concluded that a retrograde approach is favored in case of a high degree of atherosclerotic lesions around the ostium of the IA, near-occlusion of the IA and a steep angulation of the IA from the aortic arch.

Another point of interest is improving the longterm patency of the stent. A multicenter retrospective study involving 13 university hospitals in France (10) tried to determine if there is any difference between bare metal stents (BMS) and balloon-expandable covered stents (BECS) in the treatment of IA atheromatous lesions. With data collected from 93 patients treated over a 10-year period, they observed that restenosis occurred more frequently with BMS patients (RR 6.9, 95\% CI 2.2 to $22.2, p=0.001)$, the same being true for re-interventions (RR 14.6, 95\% CI 1.8 to $120.9, \mathrm{p}=0.004$ ), favoring also covered stents. The same conclusion

\section{REFERENCES}

1. Wrotniak $L$ et al. Resolution of ischemic symptoms after percutaneous angioplasty for a symptomatic subclavian artery stenosis. Journal of Vascular Surgery 2016, 64(3): 684-691.

2. Berguer R, Morasch MD, Kline RA. Transthoracic repair of innominate and common carotid artery disease: Immediate and long-term outcome for 100 consecutive surgical reconstructions Journal of Vascular Surgery 1998, 27(1): 34-42.

3. Ligush J. Innominate artery occlusive disease : Management with central reconstructive techniques. Surgery 1997, 121(5): 556-562.

4. Writing Group, Naylor AR, Ricco JB et al. Editor's Choice Management of Atherosclerotic Carotid and Vertebral Artery Disease: 2017 Clinical Practice Guidelines of the European Society for Vascular Surgery (ESVS). Eur J Vasc Endovasc Surg. 2018; 55(1):3-81.

5. Van De Weijer MAJ et al. Technical and Clinical Success and Long-Term Durability of Endovascular Treatment for Atherosclerotic was reached in the COBEST trial for the treatment of aorto-iliac occlusive disease.

Endovascular treatment of the IA remains challenging due to its large diameter, short length, bifurcation pattern into the right common carotid artery and right subclavian artery, and also its origin at the level of the aortic arch. One recognized complication is represented by stent fracture, with a reported overall rate of $34 \%$. The team at Semmelweis University (11) divided 32 patients, which were recalled for fluoroscopic examination of the implanted stent, in two groups: with and without fracture. Extensive lesions were associated with an increased incidence of stent fracture (HR 5.09; $95 \% \mathrm{CI}, \mathrm{p}=0.017$ ) but the prevalence of post-procedural symptoms, in-stent restenosis did not differ significantly between the 2 groups They concluded that IA stent fractures are common but seem to have no effect on symptoms and in-stent restenosis rate.

\section{CONCLUSION}

To sum up, even though a lot of progress has been made, PTA and stenting of the IA still remains challenging mainly due to complex anatomy and the rarity of this pathology and we must keep in mind that the decision for the revascularization technique is personalized. This case presentation brings new evidence that percutaneous transluminal stenting by retrograde approach of a near-occluded IA, is safe, improves symptoms of subclavian steal syndrome and offers a high patency rate on the mid-term.

Conflict of interest: none declared Financial support: none declared

Aortic Arch Branch Origin Obstruction: Evaluation of 144 Procedures. Eur J Vasc Endovasc Surg. 2015;50(1):13-20.

6. Hüttl K, Bérczi V et al. Angioplasty of the Innominate Artery in 89 Patients : Experience over 19 Years. Cardiovascular and Interventional Radiology 2002, 25:109-114.

7. Hattum ES Van et al. Angioplasty with or without Stent Placement in the Brachiocephalic Artery : Feasible and Durable? A Retrospective Cohort Study. Journal of Vascular and Interventional Radiology 2007, 18:1088-1093.

8. Paukovits TM et al. Percutaneous Endovascular Treatment of Innominate Artery Lesions :A Single-centre Experience on 77 Lesions. European Journal of Vascular and Endovascular Surgery 2010, 40:35-43.

9. Gralla J et al. Percutaneous and Open Retrograde Endovascular Stenting of Symptomatic High-Grade Innominate Artery Stenosis: 
Technique and Follow-Up. American Journal of Neuroradiology 2011, 32:1726-31.

10. Myriam Ammi, Samir Henni et al. Lower Rate of Restenosis and Reinterventions With Covered vs Bare Metal Stents Following Innominate Artery Stenting. Journal of Endovascular Therapy 2019, 26(3):385-390.
11. Dósa $E$, Bérczi $V$ et al. High frequency of brachiocephalic trunk stent fractures does not impair clinical outcome. J Vasc Surg. 2010; 59(3):781-785. 